\title{
Coulisses
}

Revue de théâtre

25| Hiver 2002

Varia

\section{Croisement entre sciences et poésie}

\section{(2) OpenEdition \\ 12 Journals}

Édition électronique

URL : http://journals.openedition.org/coulisses/6138

DOI : $10.4000 /$ coulisses. 6138

ISSN : 2546-9460

\section{Éditeur}

Presses universitaires de Franche-Comté

\section{Édition imprimée}

Date de publication : 1 janvier 2002

Pagination : 130-131

ISBN : 2-84627-052-X

ISSN : 1150-594X

\section{Référence électronique}

"Croisement entre sciences et poésie », Coulisses [En ligne], 25 | Hiver 2002, mis en ligne le 24 octobre 2019, consulté le 15 novembre 2019. URL : http://journals.openedition.org/coulisses/6138 ; DOI :

$10.4000 /$ coulisses.6138

Ce document a été généré automatiquement le 15 novembre 2019.

Coulisses 


\section{Croisement entre sciences et poésie}

1 La présence d'Armand Gatti à la journée inaugurale de la fête de la science n'est pas le fait du hasard. Elle se situe à la conjonction d'une double rencontre, celle du Projet Aurore et du T.U.F.C., avec Armand Gatti. La réalisation commune des deux associations étudiantes universitaires marque bien le désir qui les anime de jeter un pont entre l'art et la science.

12h. Inauguration de la manifestation «La science en fête " en présence d'Armand Gatti

18h. Dialogue entre Francis Bailly (CNRS. Cristallographie) et A. Gatti : Croisement entre sciences et poésie. (Faculté des Lettres, Amphi Donzelot)

2 A. Gatti est surtout connu comme poète

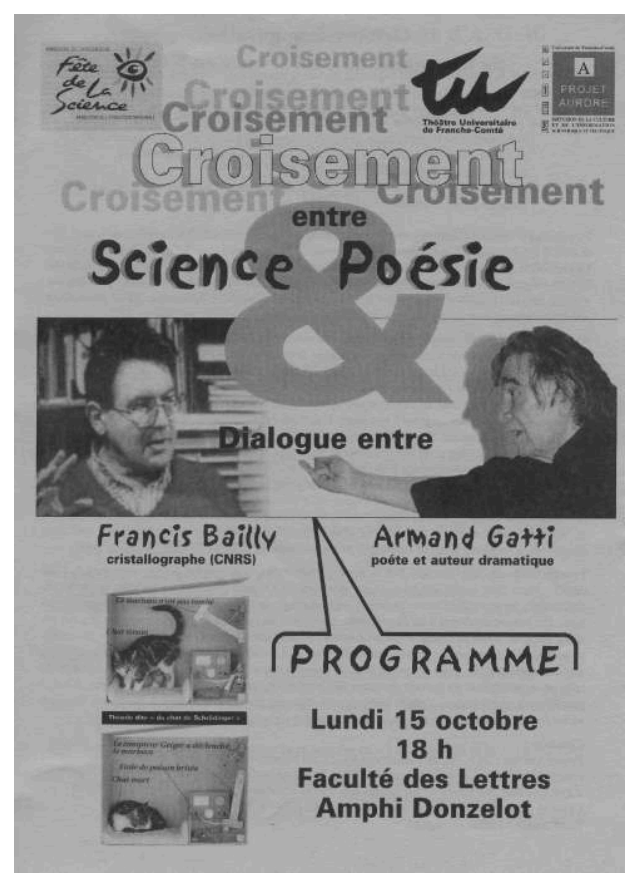
politiquement engagé aux côtés des faibles et des exploités de toutes conditions et de toutes races. Son œuvre dramatique écrit l'épopée des révoltes et révolutions de notre époque : Guatemala, Chine, Cuba, en même temps qu'elle rappelle inlassablement la barbarie innommable de l'extermination systématique. L'œuvre de Gatti interroge sans fin l'histoire pour y trouver un sens dans un entrecroisement sans cesse renouvelé des cultures du monde, chinoise, maya, occidentale des origines à nos jours. L'écriture est au centre de son projet, là où il s'agit de saisir les choses à la racine. Or une des aventures capitales de notre époque est bien celle de la science. "Interroger la science c'est immédiatement interroger la totalité d'un siècle de part en part structuré par cette pensée. Abandonnée à elle-même, à ses seuls spécialistes, sa signification, inscrite dans l'histoire, menace de disparaître... Le XX $\mathrm{XX}^{\mathrm{e}}$ siècle porte la marque des trois grands principes théoriques qu'il a découverts : le principe de relativité d'Einstein, le principe d'incertitude d'Heisenberg 
et le principe d'incomplétude de Gödel. Pour Gatti, le siècle trouve là sa véritable physionomie : relatif, incertain et incomplet. La possibilité d'un écriture poétique effective trouve ici ses nouvelles conditions. " (Stéphane Gatti in Catalogue de l'exposition les voyages de Don Quichotte, p. 68).

3 Francis Bailly est chercheur au C.N.R.S. il est spécialiste de cristallographie. Sa rencontre avec A. Gatti découle d'une lettre que le poète lui avait envoyée ainsi qu'à d'autres physiciens. "La lettre me parlait de Khlebnikov, les textes qui l'accompagnaient (en traduction) ont pour moi résonné avec mes propres questions. Un homme confronté à un ébranlement, une remise en question des connaissances et des valeurs d'une intensité, d'une radicalité, d'une profondeur rarement atteintes, confronté à un temps de révolution politique, de révolutions des connaissances (héritier de Lobatchevski, et contemporain d'Einstein), de révolution sémantique, pose devant lui la seule question qui lui paraît valide, celle du temps, ou plutôt celle de la multiplicité du concept de temps liée à celle des nombres. Et qui la pose en poète...C'est la question des physiciens, et c'est la question du judaïsme : comment l'homme, à travers ses langages va-t-il habiter le temps?» in Catalogue de l'exposition les voyages de Don Quichotte, p. 71.

\section{Rencontre-débat Armand Gatti-Francis Bailly. Fête de la Science. 15/10/01}

Quel sens donner au texte d'Armand Gatti publié dans les Cahiers de Coulisses et intitulé de façon énigmatique pour beaucoup Incertitudes de la mécanique quantique devenant chant des oiseaux du Graal pour l'entrée des groupes (de Galois) dans le langage dramatique?

5 En un dialogue fécond $\mathrm{F}$. Bailly et $\mathrm{A}$. Gatti tenteront de répondre à l'attente du public.

6 F. Bailly reprenant des éléments de sa préface éclaire les notions de physique indispensables pour rentrer dans l'univers des groupes et pour passer d'un système euclidien à un système quantique.

7 Pour A. Gatti la théorie des groupes constitue le support conceptuel par excellence lui permettant d'arriver à la poésie. Il éprouve le besoin profond d'un nouveau langage pour exprimer le monde d'aujourd'hui : la créativité du langage scientifique entrâ̂ne la créativité du langage poétique.

De nombreuses anecdotes ponctuent les interventions d'A. Gatti et s'agencent en puzzle, faisant saisir ainsi le fil rouge de la pensée gattienne.

9 La cassette enregistrée lors de cette rencontre-débat est à la disposition des personnes intéressées au bureau du T.U., université de Franche-Comté à Besançon. 